\title{
Improvement of Functional Outcome Via Supercharged End-to-Side Anterior Interosseous to Ulnar Motor Nerve Transfer: A Case Series
}

\author{
Dylan J Parker ${ }^{1,2 *}$, Robert Teixeira ${ }^{\mathbf{1}}$ and Paul A Sibley ${ }^{\mathbf{1}}$ \\ ${ }^{1}$ Department of Orthopedic Surgery, Lehigh Valley Health Network, Allentown, PA, \\ USA \\ ${ }^{2}$ USF Health Morsani College of Medicine, University of South Florida, Tampa, FL, USA \\ *Corresponding Author: Dylan J Parker, Department of Orthopedic Surgery, Lehigh \\ Valley Health Network, Allentown, PA and USF Health Morsani College of Medicine, \\ University of South Florida, Tampa, FL, USA.
}

\author{
Received: August 29, 2020 \\ Published: September 21, 2020 \\ (C) All rights are reserved by Dylan J \\ Parker., et al.
}

\begin{abstract}
Anterior interosseous to ulnar motor nerve transfer offers a viable solution in the management of high ulnar nerve injuries. Following clinical observation, we sought to determine if supercharged anterior interosseus nerve (AIN) to ulnar motor nerve transfer confers a clinically significant improvement in functional outcome as measured by the Disabilities of the Arm, Shoulder, and Hand (DASH) score. Our objective was to track the functional outcomes compared to baseline following supercharged AIN to ulnar motor nerve transfer in patients with high ulnar nerve injuries. A case series comprising a retrospective cohort included all patients who underwent an anterior interosseous to ulnar motor nerve transfer within a one-year time interval. Functional outcomes were assessed via the preoperative to postoperative change in outcome measures including DASH scores, hand strength and active range of motion. Sixteen patients were included in the final review (81.25\% male, 18.75\% female, mean age $54.81 \pm 16.26$ years) with a mean follow-up of $2.06 \pm 0.892$ months. Postoperative DASH scores for all combined injury etiologies, compressive etiologies, and traumatic etiologies decreased an average of $41.99 \%, 29.38 \%$ and $88.23 \%$ respectively. Grip strength, 3-point pinch strength, and lateral pinch strength increased an average of $41.37 \%, 16.38 \%$ and $55.84 \%$ respectively. Ulnar deviation and radial deviation increased an average of $46.38 \%$ and $31.58 \%$ respectively. Our findings demonstrate that end-to-side AIN to ulnar motor nerve transfer for ulnar nerve injuries may be a powerful adjunct to traditional management of above elbow nerve injuries.
\end{abstract}

Keywords: Anterior Interosseus Nerve (AIN); Disabilities of the Arm, Shoulder, and Hand (DASH); Ulnar Motor Nerve

\section{Introduction}

Peripheral nerve injuries pose a complex reconstructive challenge due to the inherently slow rate of native nerve regeneration and the high likelihood of incomplete functional recovery [1-3]. Such injuries result in loss of sensory/motor function, with muscle fibers undergoing irreversible changes after 12 - 18 months of denervation [1]. Functional motor recovery after peripheral nerve injury is predominantly determined by the time to motor end plate reinnervation and the absolute number of regenerated motor axons that reach the target $[1,2]$. Nerve transfers shorten the distance for axon regeneration and consequently the time for muscle reinnervation. Prior techniques to address denervation injuries such as tendon transfers do not always allow fluid motion and adequate strength [1].

High ulnar nerve injuries are of particular significance due to the ulnar nerve's critical role in intrinsic hand function, including grip and pinch strength [1]. The anterior interosseous nerve (AIN) has been the donor nerve of choice for recovering some ulnar nerve intrinsic function [2]. Traditional end-to-end coaptation has been 
shown to improve recovery of intrinsic muscle function in complete ulnar nerve injury [2,3]. For incomplete ulnar nerve injuries, the "supercharged" end-to-side motor nerve transfer has been proposed to augment functional recovery by supplementing the fascicles of the ulnar nerve. This approach relies on repurposing terminal AIN median motor fibers to the ulnar nerve in the distal forearm. Supercharged AIN to ulnar motor nerve transfer has shown promise in reversal of intrinsic muscle atrophy as well as improvement of functional outcomes [2-4]. Reports have demonstrated a significant improvement of intrinsic muscle reinnervation and clawing deformity correction with supercharged endto-end motor nerve transfer as compared to isolated ulnar nerve repair by fostering axonal growth along the new pathway $[5,6]$.

Anterior interosseous to ulnar motor nerve transfer offers a viable solution in the management of high ulnar nerve injuries. The purpose of this study is to track the functional outcomes compared to baseline following supercharged AIN to ulnar motor nerve transfer in patients with high ulnar nerve injuries - either ulnar nerve transections or severe compressive neuropathies. Specifically, we sought to determine if patients undergoing a supercharged AIN to ulnar motor nerve transfer will have a significant improvement in functional outcome as measured by the Disabilities of the Arm, Shoulder, and Hand (DASH) score.

\section{Methods}

The present study was designed as a case-series. We performed an IRB-approved retrospective review of the electronic medical records of all patients who underwent AIN to ulnar motor nerve transfer performed by the senior author, P.S., at Lehigh Valley Health Network between August 1, 2016 and August 1, 2019. All patients received standard surgical consent by the performing surgeon, P.S., at the time of consultation.

Patients were included regardless of comorbidities or mechanism of injury. The following were excluded from the study: patients without a preoperative hand therapy evaluation, adults unable to consent, patients without adequate postoperative physical therapy follow-up ( 0 postoperative physical therapy sessions), patients $<18$ years old, pregnant women, and prisoners.

All patients included had documented clinical evidence of ulnar nerve-innervated intrinsic denervation evidenced by, for example, paralysis, muscle wasting, Froment sign, clawing deformity, or conduction delay on electromyogram (EMG). Included patients dem- onstrated one or more clinical symptoms. Patients completed pre and postoperative hand therapy evaluations. All patients participated in standard postoperative upper extremity physical therapy on at least a monthly basis following the procedure. All patients had at least 6 months of follow up with the senior author.

The surgical details of AIN to ulnar motor nerve transfer was performed in a manner similar to Barbour., et al. 2012. It was performed under 3.5x loupe magnification with a tourniquet. A nerve stimulator (Checkpoint Surgical; Highland Hills, Ohio) was utilized to confirm the motor fascicle prior to end-to-side coaptation of the AIN. Concurrent release of Guyon's canal, the carpal tunnel, and the cubital tunnel with submuscular transposition were performed.

The senior author's and hand therapists' pre and postoperative notes were reviewed in the EMR for the following objective measurements: DASH or quick DASH (qDASH) scores, grip strength, active extension of the ring finger (RF) and small fingers (SF), manual muscle testing of pronation, flexor pollicis longus (FPL), and flexor digitorum profundus (FDP) and maximum adduction and abduction hand measurements. The aforementioned parameters were tested in order to monitor patient functional status, ulnar nerve benefits of the nerve transfer, and possible median nerve deficits imposed by the nerve transfer. Preoperative and postoperative DASH scores at 3 - 6 months were considered to assess any change in intrinsic hand function. The most recent score reported within this timeframe was used in the analysis. A reduction in preoperative to postoperative DASH scores by at least $15 \%$ were clinically significant in demonstrating functional improvement.

The primary outcome investigated was the change in DASH score after surgery. Secondary outcomes were the postoperative changes in remaining objective measurements obtained by the hand therapists: grip strength, active extension of the RF and SF, manual muscle testing of pronation, FPL and FDP, and maximum adduction and abduction hand measurements. In accordance to the case-series design of the study, the included patients all received AIN to ulnar motor nerve transfer and their functional outcome measures were reported and measured with respect to achieving a clinically significant postoperative outcome.

\section{Results}

A total of 20 patients were identified who underwent AIN to ulnar motor nerve transfer during the specified timeframe, with 16 patients meeting the final inclusion criteria. A total of $n=4$ patients 
were excluded from the study. Of these 4 patients, $n=1$ was excluded due to mortality in the postoperative period due to an unrelated medical cause preventing postoperative physical therapy followup and $n=3$ were excluded due to the absence of preoperative baseline qDASH scores, hand range of motion, and hand strength data in the medical record. 16 patients were included in the final analysis as presented in figure 1.

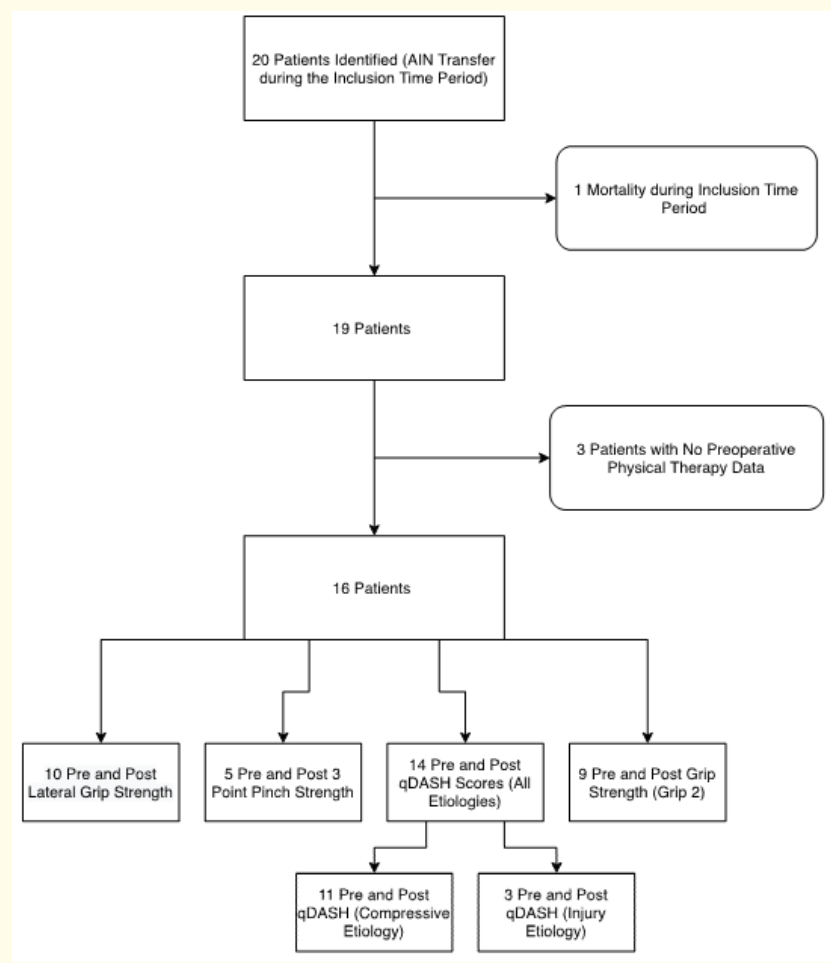

Figure 1: Flow chart of patients who met inclusion/exclusion criteria for the study population.

Demographic and comorbidity data are presented in table 1. The mean age was $54.81 \pm 16.26,13(81.25 \%)$ were male, 3 $(18.75 \%)$ were female, and $6(37.5 \%)$ underwent AIN to ulnar motor nerve transfer on their dominant hand. Average postoperative hand therapy follow-up time of 2.06 months \pm 0.892 with a range of 1.0 - 3.5 months. $10(62.5 \%)$ had preoperative comorbidities that may have compromised nerve function. Of the 16 patients, 13 $(81.25 \%)$ had a severe compressive neuropathy of nerve injury and $3(18.75 \%)$ had a traumatic (e.g. laceration) nerve injury.
14 patients had pre and postoperative qDASH scores available. qDASH scores for all combined etiologies decreased an average of 18.22 points (41.26 preoperative - 23.03 postoperative; average percentage reduction $=41.99 \%$ ) postoperatively as compared to preoperative scores. This percentage reduction was greater than the clinically significant reduction of $15 \% .10$ of the 14 patients had clinically significant reductions in qDASH scores greater than $15 \%$. The group of 14 was subsequently divided into compressive neuropathy $(n=11)$ and traumatic injury $(n=3)$ groups. The qDASH scores for the compressive neuropathy group decreased an average of 16.29 points postoperatively (43.97 preoperative - 27.67 postoperative; minimum follow-up time: 1 month; average percentage reduction $=29.38 \%$ ). 7 patients in this group experienced clinically significant reduction in scores greater than $15 \%$. The qDASH scores for the traumatic injury group decreased an average of 25.3 points (31.33 preoperative - 6.03 postoperative; average percentage reduction $=88.23 \%$ ). All 3 patients in this group experienced a clinically significant reduction in scores over $15 \%$. The above are presented in table 2 .

\begin{tabular}{|c|c|c|}
\hline \multicolumn{3}{|c|}{ Demographics } \\
\hline \multirow{2}{*}{$\operatorname{Sex}^{\mathrm{a}}$} & Male & $13(81.25)$ \\
\hline & Female & $3(18.75)$ \\
\hline Age (years) $^{\mathrm{b}}$ & & $54.81 \pm 16.26$ \\
\hline \multirow{2}{*}{ Injury side ${ }^{a}$} & Dominant Hand & $6(37.5)$ \\
\hline & Non-dominant hand & $10(62.5)$ \\
\hline $\begin{array}{l}\text { Follow-up length } \\
\text { (months) }^{\mathrm{b}}\end{array}$ & & $2.06 \pm 0.892$ \\
\hline \multirow{2}{*}{ Injury Etiology ${ }^{\mathrm{a}}$} & Compression & $13(81.25)$ \\
\hline & Trauma & $3(18.75)$ \\
\hline \multirow{5}{*}{ Comorbidities $^{\mathrm{a}}$} & Diabetes Mellitus Type II & $1(6.25)$ \\
\hline & Neuropathy & $3(18.75)$ \\
\hline & Hypothyroid & $1(6.25)$ \\
\hline & Cervical Spinal Stenosis & $1(6.25)$ \\
\hline & $\begin{array}{c}\text { Diabetes Mellitus Type II + } \\
\text { Neuropathy }\end{array}$ & $4(25)$ \\
\hline
\end{tabular}

Table 1: Demographic, preoperative, and postoperative data $(n=16)$.

${ }^{\mathrm{a}} \mathrm{n}$ (percent).

${ }^{\mathrm{b}}$ Mean \pm standard deviation. 


\begin{tabular}{|l|c|c|c|}
\hline & qDASH All Etiologies & qDASH Compressive Etiology & qDASH Traumatic Etiology \\
\hline Mean Difference $^{\mathrm{b}}$ (Pre - Post) $^{\mathrm{c}}$ & $18.22 \pm 8.82(41.26-23.03)$ & $16.29 \pm 10.72(43.97-27.67)$ & $25.30 \pm 30.08(31.33-6.03)$ \\
\hline No. of Patients & 14 & 11 & 3 \\
\hline Minimum & -11 & -11 & 16 \\
\hline Maximum & 39 & 39 & 39 \\
\hline Median & 17 & 14 & 20.91 \\
\hline Mean Reduction $^{\mathrm{d}}$ & 41.9 & 29.38 & 88.23 \\
\hline $\begin{array}{l}\text { No. of Patients Achieving Clini- } \\
\text { cally Significant Reduction }^{\mathrm{e}}\end{array}$ & 10 & 7 & 3 \\
\hline
\end{tabular}

Table 2: Preoperative to postoperative difference in qDASH scores.

${ }^{\mathrm{b}}$ Mean \pm standard deviation.

${ }^{c}$ (Preoperative mean - postoperative mean).

${ }^{\mathrm{d}}$ Percent.

${ }^{e}$ Clinically significant reduction: $>15 \%$.

qDASH: quick Disabilities of the Arm, Shoulder, and Hand score.

Postoperatively, of the patients with pre and postoperative grip strength $(\mathrm{n}=9)$ data available, grip strength increased an average of $10.88 \mathrm{lbs}$ (average percentage increase $=41.37 \%$ ). Of the patients with pre and postoperative lateral pinch strength $(n=9)$ and 3 -point pinch strength $(n=5)$ data available, lateral pinch strength increased an average of 1.34 lbs (average percentage increase = $16.38 \%$ ) and 3-point pinch strength increased an average of 2.14 lbs (average percentage increase $=55.84 \%$ ). Table 3 details the above. Mean data for changes in ulnar deviation and radial deviation can be found table 4 .

\begin{tabular}{|l|c|c|c|}
\hline & Grip Strength & Lateral Pinch & $\begin{array}{c}\text { 3 Point } \\
\text { Pinch }\end{array}$ \\
\hline Mean $^{\mathrm{b}}$ & $10.88 \pm 9.92$ & $1.34 \pm 1.68$ & $2.14 \pm 1.85$ \\
\hline No. of Patients & 9 & 10 & 5 \\
\hline Minimum & -7 & -3 & 0 \\
\hline Maximum & 38 & 4 & 3.6 \\
\hline Median $^{\text {Mean Increase }}$ & 12 & 1.6 & 2.9 \\
\hline c $^{\mathrm{s}}$ & 41.37 & 16.38 & 55.84 \\
\hline
\end{tabular}

Table 3: Preoperative to postoperative difference in hand strength.

${ }^{\mathrm{b}}$ Mean \pm standard deviation.

cPercent.

\begin{tabular}{|c|c|c|}
\hline & Ulnar Deviation & Radial Deviation \\
\hline Mean $^{\mathrm{b}}$ & $7.2 \pm 11.46$ & $1.0 \pm 15.66$ \\
\hline No. of Patients & 5 & 5 \\
\hline Minimum & 0 & -20 \\
\hline Maximum & 20 & 13 \\
\hline Median & 2 & 5 \\
\hline Mean Increase $^{\mathrm{c}}$ & 46.38 & 31.58 \\
\hline
\end{tabular}

Table 4: Preoperative to postoperative difference in ulnar and radial deviation.

bMean \pm standard deviation.

cPercent.

\section{Discussion}

Our study details the senior author's functional outcomes performing end-to-side AIN to ulnar motor nerve transfers for ulnar nerve injuries. Functional outcomes of patients with both compressive (severe cubital tunnel syndrome) and traumatic (above-elbow ulnar nerve laceration) etiologies were considered and compared. A clinically significant improvement, as measured by an improvement in their DASH scores, was noted in both patient groups. We examined additional objective data and found a dramatic improvement in grip and 3-point pinch strengths. Improvements in exten- 
sion about the ring and small finger MCP and PIP joints as well as radial and ulnar deviation, while generally positive, were less predictable.

Traditional management of severe ulnar nerve compression or high ulnar nerve injuries often had poor functional outcomes due to a slow rate of nerve recovery paired with a long distance from injured nerve end to motor plate, leading to an incomplete functional recovery $[1,2]$. Side-to-end nerve transfer acts as a baby-sitter procedure by decreasing the distance and time for motor reinnervation for the donor nerve while awaiting the native nerve fibers to regenerate. Our findings demonstrate that end-to-side AIN-to-ulnar motor nerve transfer for ulnar nerve injuries may be a powerful adjunct to traditional management of above elbow nerve injuries. Our findings are consistent with other studies [24,7-11].

The study had several limitations. It was a retrospective case series, had limited patient numbers, incomplete patient data and selection bias. Preoperative data was not available on all patients with high ulnar nerve lacerations and these patients were excluded from analysis. Strength and range-of-motion data was inconsistent between patients evaluated. Further prospective, randomized controlled trials should be performed to better characterize the benefits and potential pitfalls of end-to-side AIN to ulnar motor nerve transfers.

The demographic data is a representation of the patients requiring and consenting to end-to-side AIN to ulnar motor nerve transfers for ulnar nerve injuries during the one-year time inclusion period in the senior author's clinic. While there is a predominance of male patients (81.25\%) in the study, this was not intentional or altered. It is an accurate representation of the clinical population at this institution. The disproportion in gender in relation to high ulnar motor nerve injury observed in this study may be basis for future clinical study.

\section{Conclusion}

In conclusion, we demonstrate a clinically significant improvement in functional outcomes and intrinsic hand function after endto-side AIN to ulnar motor nerve transfers for ulnar nerve injuries within a short follow-up interval. It is a power adjunct in the management of patients with a traditionally poor outcome. The results suggest a viable surgical augmentation of functional improvement for patients with high ulnar nerve injuries. Based on observation of clinical improvement of the included patients undergoing AIN to ulnar motor nerve transfers, we hypothesize that end-to-side AIN to ulnar motor nerve transfers may lead to a quicker and overall increased functional recovery as compared to treatments without such intervention.

\section{Financial Disclosures}

None.

\section{Conflicts of Interest}

None of the authors have a financial interest in any of the products, devices, or drugs mentioned in this manuscript.

\section{Institutional Review Board (IRB) Approval}

This study was approved by the Lehigh Valley Health Network Research Ethics Board (Identification Code: IRB00001409 registered under FWA \#00000624; IRB ID: STUDY00000235). All procedures followed were in accordance with the ethical standards of the responsible committee on human experimentation (institutional and national).

\section{Bibliography}

1. Sassu P., et al. "Nerve transfers of the forearm and hand: A review of current indications". Plastic and Aesthetic Research 2.4 (2015): 195.

2. Barbour J., et al. "Supercharged End-to-Side Anterior Interosseous to Ulnar Motor Nerve Transfer for Intrinsic Musculature Reinnervation". The Journal of Hand Surgery 37.10 (2012): 2150-2159.

3. Baltzer H., et al. "Comparison of Ulnar Intrinsic Function following Supercharge End-to-Side Anterior Interosseous-to-Ulnar Motor Nerve Transfer". Plastic and Reconstructive Surgery 138.6 (2016): 1264-1272.

4. Davidge KM and Mackinnon SE. "The Supercharge End-to-Side Anterior Interosseous to Ulnar Motor Nerve Transfer for Restoring Intrinsic Function: Clinical Experience". The Journal of Hand Surgery 38.10 (2013).

5. Koriem E., et al. "Comparison Between Supercharged Ulnar Nerve Repair by Anterior Interosseous Nerve Transfer and Isolated Ulnar Nerve Repair in Proximal Ulnar Nerve Injuries". The Journal of Hand Surgery American 45.2 (2020): 104-110.

6. Jarvie G., et al. "Supercharge End-to-Side Anterior Interosseous Nerve to Ulnar Motor Nerve Transfer for Severe Ulnar Neuropathy: Two Cases Suggesting Recovery Secondary to Nerve Transfer". Journal of Orthopaedic Case Reports 8.5 (2018): 25-28. 
7. Head LK., et al. "Evaluation of intrinsic hand musculature reinnervation following supercharge end-to-side anterior interosseous to ulnar motor nerve transfer". Plastic and Reconstructive Surgery (2020).

8. Bertelli JA. "Prior to Repair Functional Deficits in Above- and Below-Elbow Ulnar Nerve Injury". The Journal of Hand Surgery (2020).

9. Delclaux S., et al. "Case report: Double nerve transfer of the anterior and posterior interosseous nerves to treat a high ulnar nerve defect at the elbow". Chirurgie de la Main 33.5 (2014): 320-324.

10. Kapoor A., et al. "Early rehabilitation of victims of traumatic ulnar injury using tendon transfer". Injury (2020).

11. Namazi H and Haji Vandi S. "Supinator to ulnar nerve transfer via in situ anterior interosseous nerve bridge to restore intrinsic muscle function in combined proximal median and ulnar nerve injury: a novel cadaveric study". The Journal of Surgical Research 211 (2017): 95-99.

\section{Assets from publication with us}

- Prompt Acknowledgement after receiving the article

- Thorough Double blinded peer review

- Rapid Publication

- Issue of Publication Certificate

- High visibility of your Published work

Website: https://www.actascientific.com/

Submit Article: $\underline{\text { https://www.actascientific.com/submission.php }}$

Email us: editor@actascientific.com

Contact us: +919182824667 